\title{
Exploiting Node Localization for Performance Improvement of Vehicular Delay-Tolerant Networks
}

\author{
Vasco N. G. J. Soares ${ }^{1,2,3}$, Joel J. P. C. Rodrigues ${ }^{1,2}$, Farid Farahmand ${ }^{4}$, and Mieso Denko ${ }^{5}$ \\ ${ }^{1}$ Instituto de Telecomunicações, Portugal \\ ${ }^{2}$ Department of Informatics, University of Beira Interior, Covilhã, Portugal \\ ${ }^{3}$ Superior School of Technology, Polytechnic Institute of Castelo Branco, Castelo Branco, Portugal \\ ${ }^{4}$ Department of Engineering Science, Sonoma State University, CA, USA \\ ${ }^{5}$ Department of Computing and Information Science, University of Guelph, Guelph, Ontario, Canada \\ vasco.g.soares@ieee.org; joeljr@ieee.org; farid.farahmand@sonoma.edu; denko@cis.uoguelph.ca
}

\begin{abstract}
Vehicular Delay-Tolerant Networks (VDTNs) are characterized by high node mobility, intermittent connectivity, and short contact durations. Such factors cause incomplete transmissions and the waste of link capacity. To address these issues, this paper explores the use of node localization in VDTNs. The exchange of signaling information related to nodes' real-time location, current trajectory, velocity, and transmit range allows a Contact Prediction Algorithm to estimate contact durations. This information can be used in conjunction with additional signaling information (e.g. link data rate), to determine the maximum number of bytes that can be transmitted during contact opportunities. A Contact Duration Scheduling Policy can use this information to prevent incomplete transmissions, while increasing the number of successfully relayed bundles and improving data link utilization. Through a simulation study, we investigate the benefits of introducing the concept of node localization, and evaluate the performance of the proposed Contact Prediction Algorithm and Contact Duration Scheduling Policy. We demonstrate the gains introduced by this approach in comparison with an environment where VDTN nodes have no access to localization information.
\end{abstract}

Index Terms - Vehicular Delay-Tolerant Networks; Signaling; Localization Information; Contact Duration Prediction; Scheduling Policies; Performance Assessment

\section{INTRODUCTION}

Vehicular delay-tolerant networks (VDTNs) [1] are a promising technology for vehicular communications. In VDTNs, the movement of vehicles and their bundle relaying service is used to enable network connectivity under unreliable conditions, creating application scenarios for nonreal time services.

VDTNs are based on the delay-tolerant networking (DTN) paradigm [2]. Nevertheless, VDTN is a new proposal with a layered architecture that assumes the separation between the control and the data planes [1]. The control plane is responsible for signaling and resources reservation. To perform these tasks, it exchanges control information related to network nodes' type, energy and buffer constraints, supported link technologies, data rates, and transmit ranges. The control plane uses this information for data plane setup, activation, control, and deactivation, in order to optimize data bundle transmission, performed at the data plane level.

In addition, signaling information can be carried out-of-band using a low-powered, long-range, low bandwidth link. Whereas data bundles can be exchanged using a high-powered, short-range, and high bandwidth link connection. The separation between control and data planes allows both planes to evolve independently. New specifications, functions and activities can be deployed at one of these planes, without having to change the other one. This work builds on these concepts to address the problems of transmitting data bundles over short-lived links, which are well known characteristic of VDTNs due to high node mobility, intermittent connectivity, low node density and opportunistic contacts.

The paper proposes a new important function at the control plane, named node localization. It allows network nodes to exchange control information related to their geographical location, current path and velocity. In this sense, a Contact Prediction algorithm (CP) to estimate contact durations, predicting the maximum number of bytes that is possible to transmit at contact opportunities is proposed. Furthermore, a Contact Duration scheduling policy (CD) is also presented. This policy selects the data bundles (for transmission) that do not exceed the number of bytes indicated by the Contact Prediction Algorithm.

To evaluate the impact of node localization on the overall network performance, a set of metrics is considered. In this regard, data plane link bandwidth utilization, bundles delivery ratio, and bundles average delay are studied.

The remainder of this paper is organized as follows. Section II identifies the problem and presents our contribution based on real-time node localization. Section III describes our approach focusing on a contact prediction algorithm and contact duration scheduling policy. Its performance evaluation, presenting the simulation scenario and results analysis, is discussed in Section IV. Section V concludes the paper and points out future research directions. 


\section{Problem Description}

During a contact opportunity, if VDTN network nodes are not aware of the contact duration, they may start to transmit data bundles whose size exceeds the maximum number of bytes that can be transmitted before the link is terminated. This results in incomplete bundle transmissions, as illustrated in Figure 1. We assume that reactive fragmentation [3] is not allowed. Hence, the partially transferred bundles are discarded, causing the waste of data link capacity and of energy resources.

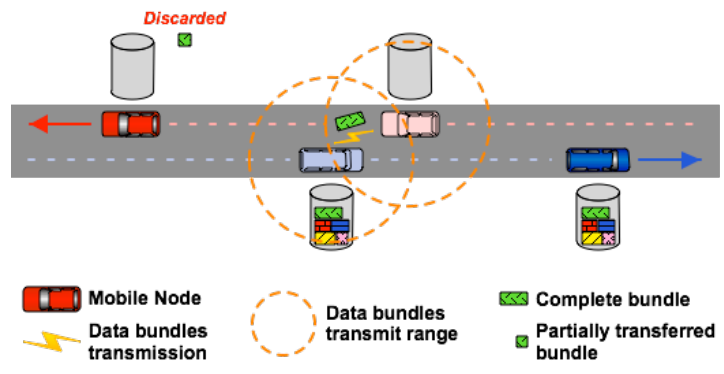

Fig. 1. Illustration of an incomplete bundle transmission.

The main idea to solve this problem can be described as follows. Assuming that network nodes have access to information about their real-time geographical location, current path and velocity, the objective is to exchange this information as signaling data and use it to predict the contact period between nodes. It defines the beginning and end times of nodes' contact. Contact duration can also be used in conjunction with information about the data link bandwidth, to determine the maximum number of bytes that can be transmitted during a contact opportunity.

Intuitively, the access to this information allows the improvement and optimization of bandwidth utilization in the data plane connections increasing the number of successfully relayed data bundles. This contributes to enlarge bundles delivery probability and decrease bundles average delay. Next section describes this proposal in detail.

\section{Contact Prediction Algorithm AND Contact DURATION SCHEDULING POLICY}

The proposal of a contact prediction algorithm (CP) assumes that network nodes have access to a location service based on position information derived using global positioning system (GPS) or other similar system. It also introduces the following new node attributes to be included on control messages: realtime geographical location, current path, and instant velocity.

The CP algorithm uses this additional signaling data in conjunction with map information to predict the topology holes without connectivity that might exist due to the spatial constraints of node movement. CP estimates the period of time where nodes will be able to exchange data bundles. It is defined as the contact duration time between nodes. Based on this information and the link data rate (data plane), the estimation of the maximum number of bytes that can be transmitted during a contact opportunity is obtained. Again, using this maximum number of bytes that can be transmitted in conjunction with the remaining signaling information, enables the decision module (shown in Figure 2) to determine whether to ignore or accept the contact opportunity. This contact opportunity may be ignored if nodes have energy or buffer constraints, or if the predicted contact duration is too short for a successful bundle transmission.

When the decision module accepts a contact opportunity, the control information is used to setup, activate, control and deactivate the data plane connection. The contact duration time is used to determine when the data link should be activated and deactivated. This contributes to reduce the power consumption of this high-powered and high bandwidth link.

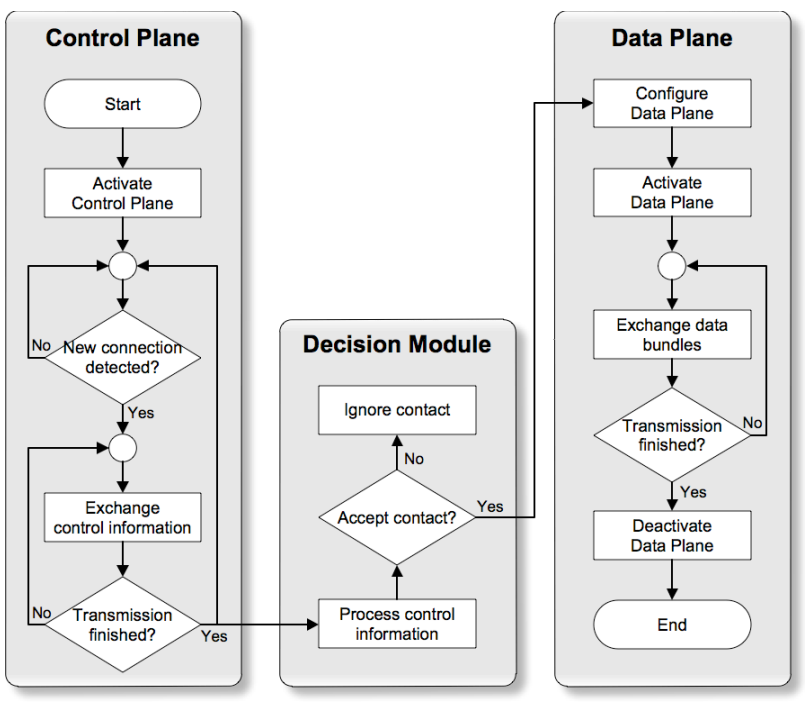

Fig. 2. Flowchart describing control plane and data plane interaction, coordinated by the decision module.

In order to improve the data link bandwidth utilization, a contact duration scheduling policy (CD) should be enforced. This policy selects the bundles to be forwarded at a contact opportunity using a double criterion. CD uses the information provided by the contact prediction algorithm to schedule (a set of) bundles whose size does not exceed the estimated maximum number of bytes that can be transmitted during a contact opportunity. In addition, bundles with longer remaining time-tolive (TTLs) are scheduled to be sent first, since this decreases their average delay while increasing their delivery ratio [4]. TTL is a timeout value that, when expires, expresses that bundles should be discarded from nodes' buffers, since they are no longer meaningful. In cases of buffer overflow, a lifetimebased drop policy discards the bundles with lowest remaining TTLs first.

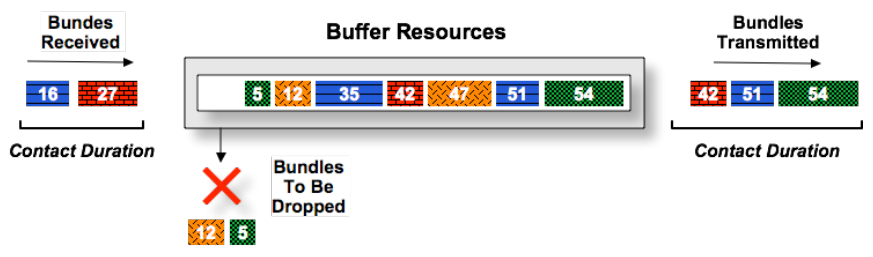

54 Data Bundle (with remaining time-to-live of 54 minutes)

Fig. 3. Contact duration scheduling policy and lifetime-based drop policy.

Figure 3 illustrates the operation principle of $\mathrm{CD}$ and lifetime-based drop policies. As may be observed, during a 
contact opportunity, the network node uses the predicted contact duration to decide to transfer the bundle with a remaining TTL of 42 minutes instead of the bundle with 47 minutes. It takes into account the bundle size, where the scheduling of the bundle with 47 minutes would result in an incomplete data transmission and in its discarding at the receiving node. The remaining contact duration is insufficient for scheduling another bundle.

Figure 4 briefly illustrates the relations between the above concepts. While moving along its path, a mobile node (e.g. a vehicle) uses its control plane connection to detect the presence of another network node and to discover its characteristics. The mobile node receives the information that the peer node is a stationary relay node. Relay nodes are fixed devices located at road intersections that increase the number of contact opportunities and the message delivery probability, as shown in $[5,6]$. Furthermore, the exchanged control information related to nodes' localization allows these nodes to determine the period of time where their data plane should be in the active state and what data bundles should be transmitted. A connection between two mobile nodes or any other type of VDTN nodes follows the same process.

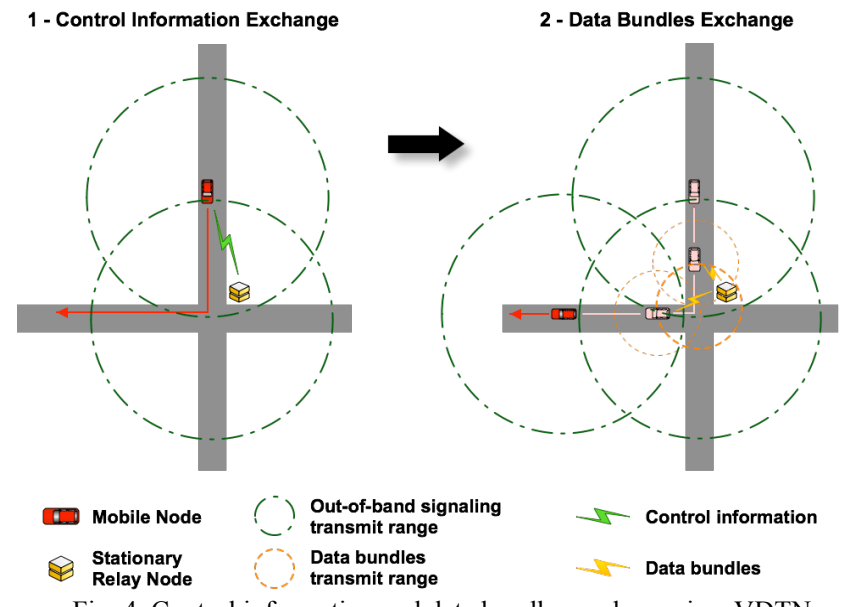

Fig. 4. Control information and data bundles exchange in a VDTN.

\section{PERformance Evaluation}

This section studies the effect of node localization on the performance of a vehicular delay-tolerant network. The study is driven through simulation and a simulator based on the Opportunistic Network Environment (ONE) simulator [7] was developed. The changes on ONE includes the implementation of the VDTN layered architecture model proposed in [1] and the construction of the above-described contact prediction (CP) algorithm and the contact duration (CD) scheduling policy.

We are particularly interested to evaluate the reliability and efficiency of the proposed contact prediction algorithm and to analyze the performance of a VDTN when the new contact duration scheduling policy is enforced. The performance metrics considered for the $\mathrm{CP}$ algorithm are the number of predicted contacts and the estimated contact duration. The CD policy is evaluated in terms of aborted bundles transmission, bundle delivery probability, bundle average delay, and routing protocol overhead ratio.
Next subsections describe the simulation scenario and the corresponding results analysis.

\section{A. Network Setup}

The simulation scenario assumes the simulation of a period of 12 hours (e.g., from 8:00 to 20:00). We consider 40 vehicles moving on roads of a map-based model of Helsinki downtown area shown in Figure 5. Vehicles have a 100 Mbytes message buffer. They move between random locations with random pause times between 5 and 15 minutes. To create scenarios with different contact durations, vehicles' speed changes between $20,30,40$, and $50 \mathrm{~km} / \mathrm{h}$, across the simulations.

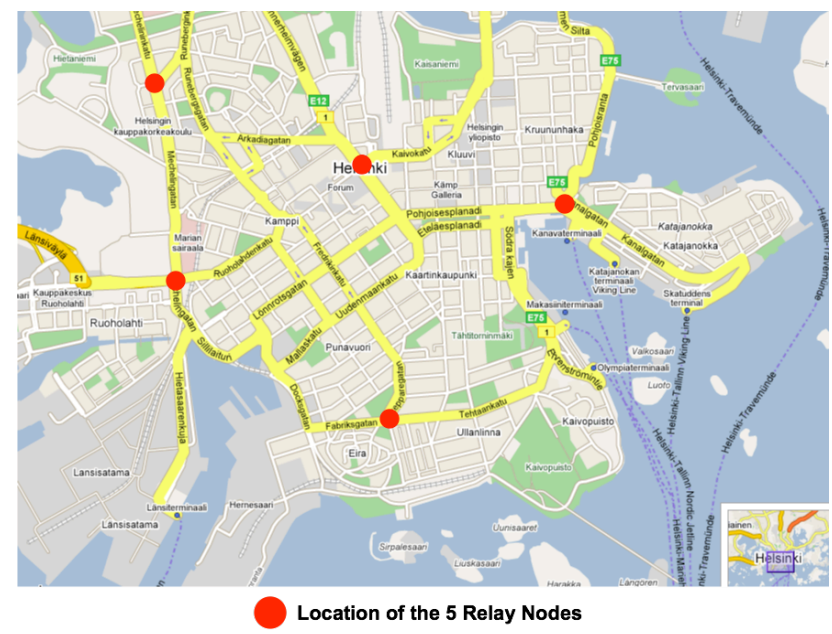

Fig. 5. Helsinki simulation scenario (area of $4500 \times 3400$ meters).

A set of 5 stationary relay nodes was placed at road intersections, each one with a 500 Mbytes message buffer (identified in Figure 5). To generate data bundles with different sizes (representing traffic created by different VDTN applications), three event generators are considered. Each one generates bundles with sizes uniformly distributed in the ranges of $[25 \mathrm{~KB}, 100 \mathrm{~KB}],[250 \mathrm{~KB}, 500 \mathrm{~KB}]$, and $[750 \mathrm{~KB}$, $1 \mathrm{MB}]$ (Bytes) respectively. All event generators assume an inter-bundle creation interval time in a range (uniformly distributed) of $[15,30]$ seconds.

Data bundles have random source and destination vehicles, and a time-to-live (TTL) of 60 minutes. They are discarded when buffer congestion occurs or TTL expires. Network nodes exchange signaling information (out-of-band), using a link connection with an omni-directional transmission range of 90 meters. Data bundles are transmitted through a link with a data rate of $6 \mathrm{Mbps}$ and an omni-directional transmission range of 30 meters (using the standard IEEE 802.11b).

Spray and Wait [8] (binary variant) is used as the underlying DTN bundle routing scheme. Spray and Wait creates a number of copies $N$ to be transmitted ("sprayed") per message (assuming 12, in this study). Any node $A$ that has more than 1 message copies and encounters any other node $B$ that does not have a copy, forwards to $B N / 2$ message copies and keeps the rest of the messages. A node with 1 copy left, only forwards it to the final destination. 


\section{B. Performance Analysis of Contact Prediction Algorithm}

The performance analysis starts with evaluation of the behavior of the contact prediction algorithm (CP). As may be observed in Figure 6, the current version of $\mathrm{CP}$ algorithm is not able to provide a prediction for all contacts registered during the simulation period. In fact, there are some contact opportunities whose duration cannot be predicted, such as the case where a mobile node stops in the range of another stopped mobile node or in the range of a stationary relay node. We assume that, in such cases, it is not possible to estimate contact duration. Therefore, in these special cases, contact duration (CD) scheduling policy will only apply the TTL criterion to decide the order of bundles to be forwarded at that contact opportunity, possibly resulting in aborted bundle transmissions. This will be discussed in next subsection.

Furthermore, it can also be concluded that $\mathrm{CP}$ algorithm presents approximately the same difference between the average number of registered contacts and the average number of successful predicted contacts, irrespective of vehicles speed.

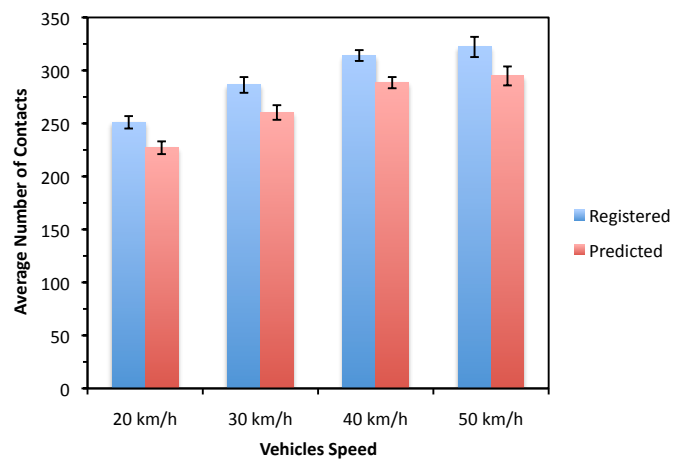

Fig. 6. Average number of contacts between network nodes as function of vehicles speed.

In respect to the accuracy of contact duration estimation, Figure 7 shows that there is a very slight difference between the $\mathrm{CP}$ predicted average contact duration and the real average contact duration (registered at the end of contact opportunities by the simulator).

As expected, Figures 6 and 7 also show that raising the vehicles velocity increases the average number of contacts registered between network nodes and, at the same time, decreases the average contact duration.

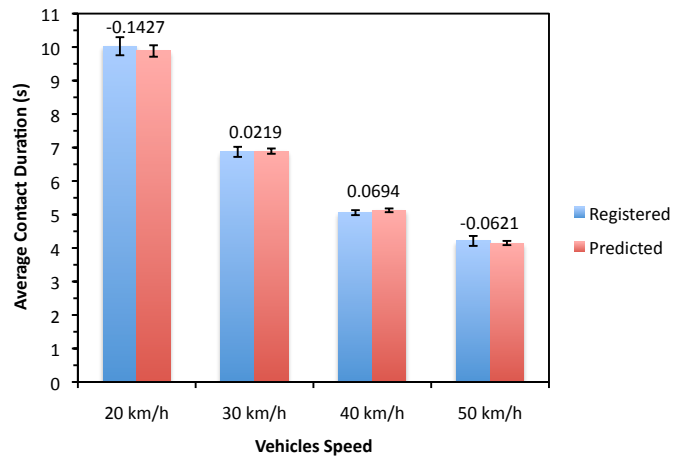

Fig. 7. Average contact duration as function of vehicles speed.

\section{Performance Analysis of Contact Duration Scheduling Policy}

In this subsection, the performance of the contact duration (CD) scheduling policy is analyzed. In order to compare this approach, a FIFO scheduling policy (first-come, first-served basis) with a "drop head" drop policy is used. These policies are enforced on Spray and Wait routing scheme.

The CD scheduling policy uses the predictions about contact durations (the maximum number of transferable bytes), provided per contact opportunity by $\mathrm{CP}$ and the information about bundles TTLs to decide what bundles should be forwarded. On the contrary, FIFO schedules messages based on the information about their receiving time (at nodes' buffers), irrespective of their size.

As expected, Figure 8 shows that the number of aborted bundle transmissions for FIFO policy is great than CD policy. Based of its working principle, FIFO is not able to avoid the problem of scheduling bundles whose size exceeds contact opportunities. A link can break during a bundle transfer resulting in an incomplete transmission. As a consequence, the partial bundle is discarded, wasting the link capacity.

As observed in the previous subsection, vehicles speed has a direct effect over the number of contacts registered during the simulation period and their measured duration. Figure 8 also allows concluding that increasing vehicles speed results in a worse performance for both scheduling policies. This is more evident for FIFO.

It is important to notice that difference between the results registered by these scheduling policies would be aggravated, if $\mathrm{CP}$ were able to provide a prediction for all contacts. Moreover, an accurate prediction about contact durations would allow $\mathrm{CD}$ to achieve an optimal behavior, preventing the occurrence of any aborted bundle transmission.

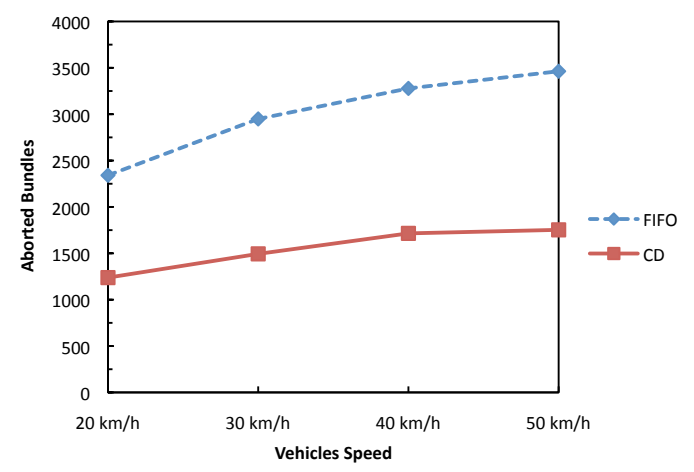

Fig. 8. Aborted bundles transmissions as function of vehicles speed.

As above-mentioned, CD policy improves data plane link utilization, increasing the number of successful bundle transmissions. Thus, network nodes store, carry, and forward more data bundles. This increases bundles probability to be successfully delivery to their final destination, as can be seen in Figure 9. Again, CD outperforms FIFO scheduling policy. Their performance differences are related to the increase of vehicles speed and to the number of aborted bundles transmissions as shown in Figure 8. 


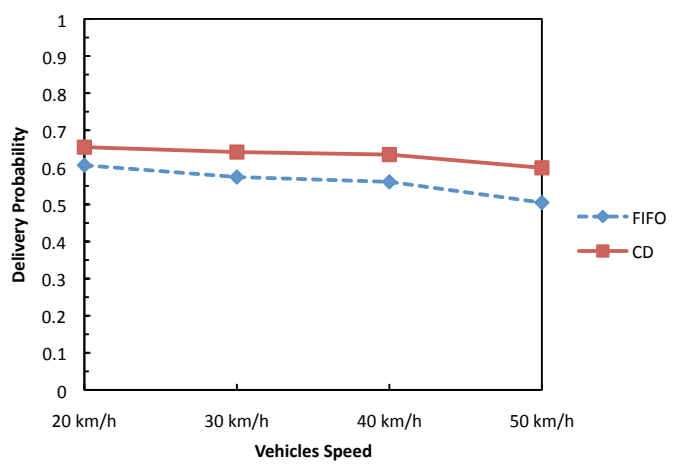

Fig. 9. Bundle delivery probability as function of vehicles speed for spray and wait routing scheme, using FIFO and CD scheduling policies.

Besides increasing bundle delivery probability, Figure 10 shows CD's additional criterion of scheduling and dropping bundles based on their remaining TTL, resulting in a decrease of the message average delay, when compared to FIFO. While FIFO policy maintains approximately the same average delay independently of the vehicles speed, $\mathrm{CD}$ average delay decreased.

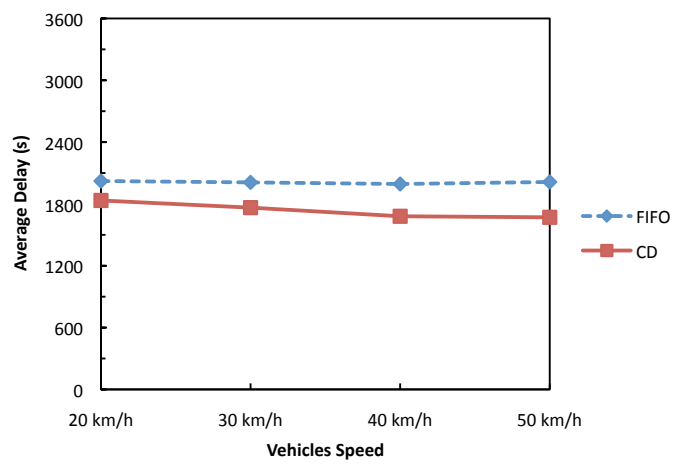

Fig. 10. Bundle average delay as function of vehicles speed for spray and wait routing scheme, using FIFO and CD scheduling policies.

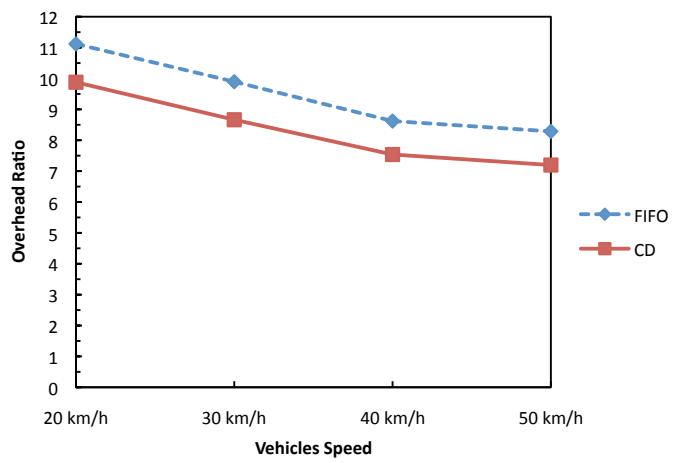

Fig. 11. Spray and Wait overhead ratio as function of vehicles speed for spray and wait routing scheme, using FIFO and CD scheduling policies.

Figure 11 shows the overhead ratio registered for Spray and Wait routing scheme, when the two scheduling policies were enforced. Overhead ratio can be defined as one measure of bandwidth efficiency of the routing protocol, since it determines how many extra successful bundle transfers were needed for each bundle delivery. As may be seen in this figure, $\mathrm{CD}$ scheduling policy also outperforms the traditional FIFO service discipline. FIFO larger overhead ratio will also increase contention for resources in the network (e.g. buffer and bandwidth). Moreover, the differences between overhead ratios registered for these policies were not aggravated when vehicles speed increased.

\section{CONClusions AND Future WORK}

This paper addressed the use of node localization information in vehicular delay-tolerant networks (VDTN). The motivation for this work comes from the need to be able to predict contact durations between network nodes, in order to cope up with short-lived links, avoiding incomplete bundle transmissions. To this effect, a contact prediction (CP) algorithm and a contact duration (CD) scheduling policy were proposed. The CP algorithm processes localization-signaling data to predict contact duration while the $\mathrm{CD}$ policy uses information about contact duration to determine which bundles should be transmitted during a data exchange session between nodes. The impact study of these approaches on the performance of VDTNs was conducted by simulation and good results were obtained.

For the future work, we intend to focus on the use of contact duration information to enable proactive bundle fragmentation.

\section{ACKNOWLEDGMENTS}

Part of this work has been supported by Instituto de Telecomunicações, Next Generation Networks and Applications Group (NetGNA), Portugal, in the framework of the Project VDTN@Lab, and by the Euro-NF Network of Excellence from the Seventh Framework Programme of EU.

\section{REFERENCES}

[1] V. N. G. J. Soares, F. Farahmand, and J. J. P. C. Rodrigues, "A Layered Architecture for Vehicular Delay-Tolerant Networks," in The Fourteenth IEEE Symposium on Computers and Communications (ISCC'09), Sousse, Tunisia, July 5-8, 2009.

[2] S. Burleigh, A. Hooke, L. Torgerson, K. Fall, V. Cerf, B. Durst, K. Scott, and H. Weiss, "Delay-Tolerant Networking: An Approach to Interplanetary Internet," in IEEE Communications Magazine, vol. 41, 2003, pp. 128-136.

[3] V. Cerf, S. Burleigh, A. Hooke, L. Torgerson, R. Durst, K. Scott, K. Fall, and H. Weiss, "Delay-Tolerant Networking Architecture," RFC 4838, April 2007, [Online]. Available: ftp://ftp.rfc-editor.org/innotes/rfc4838.txt.

[4] V. N. G. J. Soares, J. J. P. C. Rodrigues, P. S. Ferreira, and A. Nogueira, "Improvement of Messages Delivery Time on Vehicular Delay-Tolerant Networks," in The 38th Int. Conf. on Parallel Processing (ICPP-2009) Workshops - The Second Int. Workshop on Next Generation of Wireless and Mobile Networks (NGWMN-09), Vienna, Austria, Sept. 22-25, 2009.

[5] V. N. G. J. Soares, F. Farahmand, and J. J. P. C. Rodrigues, "Improving Vehicular Delay-Tolerant Network Performance with Relay Nodes," in 5th Euro-NGI Conference on Next Generation Internet Networks (NGI 2009), Aveiro, Portugal, July 1-3, 2009.

[6] W. Zhao, Y. Chen, M. Ammar, M. Corner, B. Levine, and E. Zegura, "Capacity Enhancement using Throwboxes in DTNs," in IEEE International Conference on Mobile Adhoc and Sensor Systems (MASS 2006), Vancouver, October 9-12, 2006.

[7] A. Keränen, J. Ott, and T. Kärkkäinen, "The ONE Simulator for DTN Protocol Evaluation," in SIMUTools'09: 2nd International Conference on Simulation Tools and Techniques, Rome, March 2-6, 2009.

[8] T. Spyropoulos, K. Psounis, and C. S. Raghavendra, "Spray and Wait: An Efficient Routing Scheme for Intermittently Connected Mobile Networks," in ACM SIGCOMM 2005 - Workshop on Delay Tolerant Networking and Related Networks (WDTN-05), Philadelphia, PA, USA, August 22-26, 2005, pp. 252 - 259. 